\title{
Abstracts of video presentations
}

\section{V1_01}

Laparoscopic bilateral transposition of the ovaries: demonstration of a technique and intraoperative events S. Tzitzimikas ${ }^{1}$, A. Karavida ${ }^{2}$, D. Ioannidou ${ }^{1}$

${ }^{1}$ Saint Luke's Hospital, Panorama, Thessaloniki, Greece,

${ }^{2}$ Prenatal and Gynecologic Diagnostic Center, Thessaloniki, Greece

Aim: To present, for educational purposes, a laparoscopic technique of ovarian transposition during surgical treatment of cervical cancer, as well as concurrent intraoperative events. Materials and methods: Between November 2004 and March 2008, four cases of laparoscopic transposition of the ovaries have been performed, in the context of surgical staging and/or treatment of squamous cell cervical cancer in reproductive age women. One of these cases is selected for presentation, due to concurrent intraoperative events such as mesosalpingian arterial bleeding, unsuccessful maneuvers of electrocoagulation resulting in inadequate hemostasis.

Results: In all cases, surgery was completed laparoscopically, without any conversion to laparotomy. Ovarian function was preserved in all cases. Ultracision and EnSeal were used during the adnexal dissection and the adnexal pedicle's preparation. The ovarian fixation to the paracolic spaces was realized with the help of a pig-tailed titan nail (Protract, Tyco), instead of suturing. The adnexal dissection and fixation to the lateral peritoneal wall, using the mesosalpinx or the ipsilateral oviduct instead of the ovary and /or its vascular pedicle, is described. A correction of vascular pedicle torsion follows. During the techniques' demonstration there is a correction of the under tension fixation above the cecum instead of a trans mesenterian approach, in order to avoid a bridge defect, and prevent post-operative ileus. An unsuccessful haemostatic maneuver of mesosalpinx's bleeding, using Ultracision ACE, EnSeal and bipolar coagulation, is also described. After the final surgical review of the abdomen, and the radiological imaging of the fixation's position, an ultrasound scan confirmed the left adnexal vascular integrity.

Conclusions: The use of the mesosalpinx or the proper oviduct for adnexal fixation to the abdominal wall using
Protract, is possibly a safer alternative during the laparoscopic ovarian transposition, and may offer better tissue viability. Bilateral ovarian transposition is recommended. The surgical technique described above seems to simplify the procedure along with time saving. In case of intraoperative vascular incidents, a post-operative ultrasound study offers an evaluation of ovarian vascular integrity.

\section{V1_02}

\section{Laparoscopically repaired vaginal evisceration after postpartum hysterectomy \\ N. Waters, S. Chachan, K. Morton, A. Kent Royal Surrey Hospital, Guildford, UK}

Introduction: Vaginal vault rupture with extrusion of the intraperitoneal contents is a rare complication following hysterectomy. In our video we present laparoscopic findings and demonstrate our laparoscopic repair technique. There is a brief literature review on the incidence of evisceration and mode of hysterectomy. The first case of evisceration was described by Hyernaux in 1864 . To date the highest incidence is associated with total laparoscopic hysterectomy. The technique to close the vaginal cuff is not different from traditional closure and usage of thermal energy is thought to be the predisposing factor (Walsh et al. 2007; Nezhat et al. 1996). Interestingly, this is the only case report of this complication associated with postpartum hysterectomy with the first postoperative intercourse as a trigger event.

Case: A 34-year-old G4P3 lady underwent emergency postpartum hysterectomy to control the postpartum haemorrhage on day 10 after vaginal delivery at 34 weeks. The postoperative recovery was uneventful and the patient was discharged on day 5 . She was asymptomatic until 3 months postpartum when there was sudden protrusion of the mass from the introitus. This was associated with abdominal pain and mild nausea, however speculum examination was not remarkable. The protrusion occurred straight after the first intercourse since the time of delivery. An EUA and laparoscopy was performed $48 \mathrm{~h}$ after admission and a complete dehiscence of vaginal vault was noted. The bowel 
and omentum was adherent to the vaginal vault. There was no haematoma or pelvic collection. The vaginal edges did not look necrotic. Laparoscopic adhesiolysis was performed, the edges were trimmed and closed with 2.0 Vicryl using intracorporeal suturing technique. The patient recovered well and advised to abstain from sexual intercourse until vaginal vault is healed and a gynaecologist has given "clearance".

Conclusion: Sudden occurrence of vaginal prolapse and abdominal pain in a young lady associated with intercourse with of recent history of hysterectomy should alert a clinician to consider vaginal evisceration. The advantages of laparoscopic repair are the ability to visualise the condition of the viscera and vaginal vault and easiness of repair.

\section{V1 03}

Laparoscopic resection of the mesh sacrocolpopexyvideodemonstration of case report

D. Struppl

Na Homolce Hospital Dept of Operative Gynaecology and Minimally Invasive Surgery, Prague, Czech Republic

Introduction: The surgery of pelvic organ prolapse is associated with development of new techniques, approaches and especially of mesh implants in prosthesis surgery. The laparoscopic mesh repair of urogenital prolapse belongs to the advanced laparoscopic surgery with commonly low complications rate. Mesh protrusion approximately in $2 \%$, postoperative urinary urgence in $4 \%$ and severe hemorrhage in ca. $1 \%$.

Methods: We present a case report of 48-year-old woman who underwent 2 years ago the laparoscopic multicompartmental mesh repair for urogenital prolapse after LAVH hystesrectomy performed in 1995. The indication for the resection of sacrocolpopexy was the presacral and pelvic pain due to periapical tissue retraction.

The sequentional video demostrates initial intraabdominal finding,the terapeutic plan and surgery scheme. Following part shows the surgery with resection of the mesh presacraly and from the apex of vagina. The surgery was without any complication and the patient without any problems.

Discussion: Our approach demonstrates the possibilities and advantages of laparoscopic approach in solving of this sporadic complication.

\section{V1_04}

Laparoscopic ureteroneocystostomy with psoas hitch L. Minelli ${ }^{1}, \underline{\text { L. Mereu }}^{1}$, P. Pomini ${ }^{1}$, G. Grosso ${ }^{2}$

${ }^{1}$ Ospedale Sacro Cuore, Negrar, Verona, Italy, ${ }^{2}$ Ospedale Pederzoli, Peschiera, Italy

Laparoscopic extravesical ureteroneocystostomy is an infrequently described technique.
We present a Video on laparoscopic ureteroneocystostomy with psoas hitch for the treatment of infiltrative ureteral endometriosis.

After eradication of deep endometriosis and resection of terminal part of the right ureter, mobilization of the bladder and psoas hitch were performed to obtain a non refluxing tension free ureter anastomosis. For performing this extravesical transperitoneal ureteral reimplantation intracorporeal suturing techniques were used.

We treated 15 patients with this technique without intraoperative or postoperative severe complications. Transperitoneal laparoscopic extravesical ureteroneocystostomy with vesicopsoas hitch seems to be safe and feasible operation for distal ureteral stricture due to endometriosis.

\section{V1_05}

\section{Laparoscopic anterior rectal resection for deeply infiltrating rectovaginal endometriosis}

J. Lo, J. English

Worthing Hospital, Worthing, UK

We describe a laparoscopic approach as a new way of excising rectal endometriosis that can be used without opening any part of the rectum intraabdominally. The video showed that the ureters were stented prior to laparoscopic surgery. This was followed by bilateral ureterolysis and radical resection of the pelvic endometriosis. The rectum was reflected off the back of cervix before dissection of the mesorectum off the rectum using a Harmonic Scalpel was carried out. The bowel was divided below the level of the disease using laparoscopic stapling device EndoGIA 60. The proximal end of the diseased bowel was exteriorised through the anterior abdominal wall through a $3 \mathrm{~cm}$ suprapubic incision. It was carefully resected above the diseased level and the anvil of the CEEA 31 was inserted and secured with sutures. This segment was then reinserted into the pelvis. Closure of the $3 \mathrm{~cm}$ incision performed prior to the next step of the operation to ensure pneumoperitoneum. The distal end of the rectal stump was reanastomosed with the using the CEEA device anally. The anvil was inserted into the distal end under direct laparoscopic vision. Testing of bowel anastomosis was carried out with air test.

V1_06

Bladder endometriosis - a remarkable resemblance in a monozygotic twin-diagnosis and excision using the harmonic scalpel

J.J. (Hans) van Beek

VieCuri, Medical centre of Northern Limburg, Venlo, Netherlands 
Introduction: The presentation is aimed to show the workup and treatment of bladder endometriosis.

Methods: A monozygotic pair of twin sisters presented with an almost identical spread of endometriosis involving deep infiltration in the bladder. The work-up existing of ultrasound, IntraVenous Pyelography, MRI-scan and diagnostic cystoscopy with laparoscopy is presented. Next the laparoscopic excision of the lesions was identical for both sisters and is summarized in a 7.5 min video.

Results: Genetic factors may play an important role in the expression and presentation of endometriosis. Bladder endometriosis is rare, estimated to occur in about one percent of the patient population. In monozygotic twins endometriosis often show resemblance but the almost identical presentation of bladder endometriosis as shown here, have not been reported before. The harmonic scalpel gave the opportunity to excise laparoscopically the lesions almost bloodless in both sisters with short hospitalization and complete recovery of the micturation pain during menstruation.

Discussion: Deep endometriosis of the bladder is a rare but distressing presentation of endometriosis that can be treated by laparoscopic excision in the hands of experienced laparoscopic surgeon.

\section{V1_07}

\section{A truly keyhole colposuspension}

A. Kent, P. Barton-Smith, N. Waters

Royal Surrey County Hospital, Guildford, Surrey, UK

Laparoscopic colposuspension has been shown to be at least equivalent to open colposuspension in terms of success in treating stress incontinence (Kitchener et al., BJOG 113 (9):1007-1013, 2006). It is still criticised in terms of operating time and hospital stay.

We would like to demonstrate a truly keyhole approach to laparoscopic colposuspension which dramatically reduces the operating time allowing operations to be carried out as a daycase or extended daycase procedures. The sutures and suturing techniques are similar to those used in the open operation for which there is comprehensive long term data. There is the added advantage in the reduction of anterior wall prolapse.

This video demonstrates the key steps of the procedure. A transperitoneal approach is utilised and two small windows (keyholes) opened directly onto the ileopectineal ligaments using the Harmonic ACE, with subsequent dissection of the paravaginal space to the 'white' of the vagina. Complete dissection of the Cave of Retzius is not required. It is important to open the thin fascial membrane covering the ligament which allows entry into the correct layer and blunt dissection of the space, aided by the intraabdominal pressure $(18 \mathrm{mmHg})$ and a10 $\mathrm{mm}$ cherry dissector. It is helpful for the surgeon to palpate the lateral fornices but this can be achieved by the assistant using a probe.

Two ethibond sutures are then placed on each side between the ileopectineal ligaments and paravaginal tissues. These are tied in turn using external throws and tensioned using a knot pusher or Babcock type forceps. The peritoneal openings are then closed with a figure of 8 vicryl tied intracorporeally.

\section{Reference}

1. Kitchener HC, Dunn G et al. Laparoscopic versus open colposuspension-results of a prospective randomised controlled trial. BJOG 2006; 113(9): 1007-13.

\section{V2 01}

\section{Laparoscopic cornuotomy using a temporary tourniquet suture and diluted vasopressin injection in interstitial pregnancy \\ D.-S. Eun, Y.-S. Choi, K.-S. Shin, J. Choi \\ Eun Hospital, Kwang-Ju, Korea, Republic of}

Objective: This study evaluated the efficiency of laparoscopic cornuotomy in interstitial pregnancy and its effects on anatomical reproductive capacity.

Design and method: This was an uncontrolled retrospective case review of eight patients with interstitial pregnancy who had undergone laparoscopic cornuotomy. Laparoscopic cornuotomy was performed using a temporary tourniquet suture and the injection of diluted vasopressin around the cornual mass. The tourniquet suture was removed completely after repairing the cornu. The uterotubal patency and cornual integrity were evaluated using a hysterosalpingogram (HSG) and magnetic resonance imaging (MRI), respectively.

Results: The estimated blood loss was $50 \pm 22 \mathrm{ml}$ (mean \pm $\mathrm{SD}$ ) and the operating time was $58 \pm 16 \mathrm{~min}$. The serum $\beta$ hCG level returned to normal range about 4 weeks or so postoperatively in all patients. There were no major postoperative complications, such as hemorrhage. Four of six patients had excellent uterotubal patency on the affected side, while HSG revealed tubal occlusion of the affected proximal cornu in two patients. There was no significant difference in cornual thickness compared with the unaffected cornu in each patient and there were no remarkable defects in the cornual contour on MRI, although there was no statistical significance owing to the small sample size ( $p=0.49$, paired $t$ test).

Conclusions: The temporary tourniquet suture is a safe and effective method for hemostasis and the suture enabled the performance of laparoscopic cornuotomy in interstitial pregnancy. We believe that cornuotomy has the advantage 
of preserving reproductive capacity over cornuostomy or cornual resection.

\section{V2_02}

\section{Ovarian ectopic pregnancy}

M. Capar

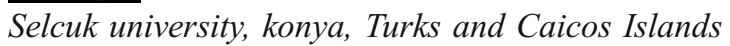

Objective: Ovarian pregnancy is a rare event. It accounts for about $1-3 \%$ of all ectopic pregnancies. Risk factors include previous pelvic inflammatory disease 'IUCD use' endometriosis and assisted reproductive technologies. Treatment of ovarian pregnancy usually requires oopherectomy or wedge resection.

Design and methods: A 32-year-old female who used IUCD for contraception, her second pregnancy proved to be ovarian ectopic pregnancy' her B-HCG level was 2322, and ultrasonographic imaging helped to put the definite diagnosis. Laparoscopic ectopic pregnancy extirpation was done by using single laparoscopic trochar entry.

Results: After 1 week of the operation B-HCG level was $<10$ ' and the patient was discharged at first postoperative day. Conclusion: Ovarian ectopic pregnancy can be treated laparoscopically with single trochar entry, and medical management with methotrexate may be an option if there is persistent trophoplastic tissue after laparoscopy. If future fertility is desired wedge resection considered. Oopherectomy should be reserved for cases of advanced gestations.

\section{V2_03}

\section{Laparoscopic promontofixation in postmenopausal patients \\ M. Visotsky, O. Kharlova \\ Moscow state university for medicine and dentistry, Moscow, Russian Federation}

Genital prolapse presents a challenge in nowadays surgery. With the introduction of new and costly techniques the old ones demand a reappraisal. The data from retrospective analysis of 15 laparoscopic promontofixations (PMF) were studied. The indications for the operation were: uterine myoma + genital prolapse (nine), genital prolapse and prolapse of cervical stump (four), prolapse of vaginal stump (two). The mean age was 57.8 \pm 7.93 , from 45 to 69 years. Reproductive function: all patients had deliveries: 1-3 (27.3\%), 2-8 (72.2\%). All patients had abortions from 2 to 8 , mean $4.5 \pm 2.1$. Three (27.3\%) patients had missed abortions from 1 to 3. The duration of postmenopause was from 1 till 23 years, mean $9.4 \pm 7.8$. The duration of prolapse- 1.5 10 years, mean $4.5 \pm 2.1$. As a first step of the operation eight patients suffered panhysterectomy, three cervical stump extirpation, three bilateral adnexectomy (patient suffered vaginal hysterectomy previously). Mesh was fixed to cervical stump in one patient. In all other patients mesh was sutured typically to levator muscles and vagina. PMF followed all operations as a second step. The duration of the operation varied from 120 to $180 \mathrm{~min}$, mean $151 \pm 23.74 \mathrm{~min}$. Mean blood loss $200 \mathrm{ml}$. Cervical stump extirpation was the most challenging part of the whole operation (105 min). Eight patients were cardiovascularily compromised. There were no intraoperative complications. Patients were discharge on seventh postoperative day. After 6 months of observation two erosions of vagina was revealed with small bleeding in one patient. This patient demanded mesh excision. One patient after cervical stump fixation required second operation because of relapse. After 6 month 12 patients estimated the postoperative results as excellent, two as satisfactory, one as unsatisfactory.

Conclusion: Laparoscopic promontofixation presents technical challenge for the surgeon but gives excellent results.

\section{V2_04}

\section{Laparoscopic myomectomy and abdominal cerclage} J. Einarsson

Brigham and Women's Hospital, Boston, MA, USA

We present a case of a concurrent laparoscopic myomectomy and laparoscopic abdominal cerclage in a patient with a history of cervical incompetence and a previously failed vaginal cerclage. We present the usage of a novel bidirectional barbed suture for the myometrial closure. The monofilament suture slides easily through tissue, but the barbs prevent any backward slippage, thereby preventing a secure closure and excellent hemostasis. We routinely use this suture for myometrial closure as well as vaginal cuff closure during a total laparoscopic hysterectomy.

\section{V2_05}

\section{Laparoscopyc lymph node debulking: indications and strategies}

A. Pessarrodona, J. Isern, J. Cassadó, J. Rodriguez, L. Codina, M. Duran

Hospital Mutua de Terrassa, Terrassa, Spain

Pelvic and/or paraaortic lymphadenectomy is considered the standard procedure to perform surgical staging of gynaecology cancer and evaluate the extent of the disease. It permits to adjust the radiotherapy and evaluate the need of chemotherapy with the minimal morbidity.

However, since some years ago, some studies conclude with enough scientific evidence the value of the lymph node dissection as a therapeutic weapon on gynaecological 
tumors: Advanced cervical cancer, endometrial cancer FIGO stage III $\mathrm{C}$ and ovarian cancer specially in those cases with macroscopically lymph node disease.

Considering this evidence, it could be established a group of indications of the pelvic/paraaortic debulking: -Locally advanced cervical cancer not subsidiary of surgery and suspicion of macroscopic lymphadenopathy.

-FIGO stage III C endometrial carcinoma

-Ovarian carcinoma

-Vulvar cancer with pelvic or inguinal lymph node infiltration.

We show in the video surgical tips and strategies to face the difficulties due to the vascular infiltration and massive nearby tissue affectation.

\section{V2_06}

\section{Videopresentation technique of anterior, posterior and total laparoscopic exenteration \\ P. Bartos, M. Skrovina, M. Trhlik \\ JG Mendel Cancer Centre, Novy Jicin, Czech Republic}

Description of videopresentation: Pelvic exenteration is a salvage procedure done in the effort to eliminate completely recurrent pelvic cancer. The primary tumors are usually those of the cervix, rectum, vagina, endometrium or urinary bladder. The latest advances in the technique involve low coloanal laparoscopic anastomosis and continent urinary diversion enabling complete or modified pelvic evisceration without the need for external appliances. The port placement is demonstrated as well as the evaluation of operability with dissection of paravesical and pararectal spaces. After appropriate lymphadenectomy the dissection continues down until the pedicle containing lateral parametrium with uterine vessels, superior haemorrhoidal vessels and ureter. The ureter is completely dissected from above the pelvic brim around psoas muscle to the ureteral canal parametrial entrance. The en bloc dissection of the neoplasm is undertaken by coagulating/ cutting instrument of laparoscopic plasmakinetic trissector. The ureter is clipped and divided at the parametrial entrance as distally as possible. In case of bladder removal the pelvic fascia is incised anteriorly, urethra identified, dissected, clipped and divided. If the colorectum must be removed the dissection continues posteriorly in the avascular plane towards the anal sphincter. The specimen is removed transperineally or vaginally. Further in the video we present side to end coloanal reanastomosis with circular stapler as well as the technique of urinary continent pouch reconstruction.

V2_07

Laparoscopic treatment of retroverted uterus associated with chronic pelvic pain

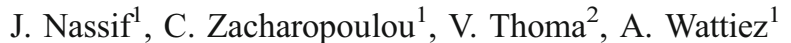

${ }^{1}$ IRCAD/EITS, Strasbourg, Bas Rhin, France, ${ }^{2}$ Hautepierre University Hospital, Strasbourg, Bas Rhin, France

Objective: Surgical treatment for pelvic congestion syndrome is still controversial, and showed failure in some data. We suggest that in patient's selection and good surgical techniques are important factor for treatment's success. We present a short video showing three operative laparoscopic techniques for the treatment of pelvic congestive syndrome. Design and methods: The three techniques are:

1. Simple round ligament plicature: its indication is distal atrophy of the round ligament associated with a normal proximal part and uterine retroversion.

2. Retrouterine round ligaments plicature: this technique is used for proximal atrophy of the round ligament with a normal distal part and for uterine retroflexion.

3. Anterior fixation of round ligaments to the aponeurosis: it is indicated in distal atrophy of the round ligament with a normal proximal part and for uterine retroversion.

Results: In a series of six patients (two patients per technique) we found that dyspareunia and chronic pelvic pain is dramatically reduced postoperatively, but there is no effect on dysmenorrhea. Pain on incision sites persists responds to minor pain killers and resolves in 10 days. No late complications occurred at three months follow-up.

Conclusion: These techniques are easy to learn and perform. Dyspareunia and chronic pelvic pain is dramatically reduced postoperatively, but there is no effect on dysmenorrhea.

\section{V3_01}

A three trocart technique for laparoscopic total and subtotal hysterectomy using Ligasure ${ }^{\circledR} 10 \mathrm{~mm}$ and the rotocut morcellator

L. Amini, M. Ketabi

Jam General Hospital, Tehran, Iran, Islamic Republic of

Objectives: Evaluation of the feasibility, safety and morbidity of a laparoscopic subtotal and total hysterectomy using Ligasure ${ }^{\circledR} 10 \mathrm{~mm}$ and the Rotocut morcellator.

Patients and methods: Our study is a series of 51 consecutive patients operated by this technique from September 2006 to march 2008. Twenty-two patients underwent supra-cervical hysterectomy and 29 patients total hysterectomy with bilateral oophorectomy. In all cases hysterectomy was indicated for dysfunctional uterine bleeding. Twenty seven patients had a former history of laparotomy (ten had one caesarean section and 17 patients had two former caesarean sections). The procedure was performed by Ligasure $^{\circledR} 10 \mathrm{~mm}$ for pedicle ligation-section and electromechanical morcellator (Rotocut; Karl Storz) to remove 
uterus from abdominal cavity in case of supra-cervical hysterectomy.

The mean operating time was 75 min (range 50 to 220). The mean blood loss was $105 \mathrm{cc}$ (60 to $310 \mathrm{cc}$ ). The mean weight of the uterus was $290 \mathrm{~g}$ (range 80 to 580). One conversion to laparotomy was performed for haemorrhage $(0.019 \%)$ and one bladder injury diagnosed and treated during surgery $(0.019 \%)$. Hospital stay was 1.18 days (from 1 to 3 days). Twenty-three active patients had 1 week off work.

Conclusion: Using a ligation-section system with the same instrument reduces time, haemorrhage and the need for multiple trocarts in this modality and decreases the total operating time which is admitted to be the disadvantage of the laparoscopic route comparing to the vaginal route. Using electromechanical rotocut morcellator allows quick removal of enlarged uteri by laparoscopy in cases where vaginal route is usually contra-indicated.

\section{V3_02}

The Plymouth colpotomy tube and uterine manipulator J. Frappell, D. Barclay, M. Norbrook

Derriford Hospital, Plymouth, UK

Background: We have previously reported on the use of the Plymouth colpotomy tube for total laparoscopic hysterectomy (TLH). We developed this as an alternative to similar devices currently available which we felt were unnecessarily complicated, and whose cost was a disincentive to uptake of the TLH procedure (ESGE 15th Annual Congress Oct 2006). We have recently developed a simple uterine manipulator which can be used with the colpotomy tube during TLH procedures.

Aim: To demonstrate both the Plymouth colpotomy tube and uterine manipulator.

Method: This video presentation describes the colpotomy tube and uterine manipulator and demonstrates their use during a total laparoscopic hysterectomy.

Results: The Plymouth colpotomy tube (LINA UK) has proved highly effective in the delineating of the vaginal fornices and providing a gas-tight seal during TLH. Its use can also be applied to other procedures involving colpotomy such as laparoscopic excision of endometriotic nodules involving the vaginal vault

The combined use of the Plymouth colpotomy tube and uterine manipulator has been a significant advance in our ability to perform the TLH more safely and with greater efficiency. Its use with the colpotomy tube has not significantly increased the overall cost of the device.

Conclusion: The uterine manipulator combined with the Plymouth colpotomy tube has improved operative performance.
V3 03

Transabdominal laparoscopic cerclage at 14th week of pregnancy

J. Isern, A. Pessarrodona, J. Cassadó, X. Urquizu, J. Rodriguez

Hospital Mutua De Terrassa, Terrassa, Spain

Indications of the transabdominal cervicoithsmic cerclage are the previous failure of vaginal cerclage, extremely short cervix due to previous cervical surgery, exposition to dietyletilbestrol or Müllerian alterations and cervical damage caused by obstetric traumatism.

Video: We present a case of a first trimester pregnant women with previous four second trimester miscarriage (inefectives shydokar cerclages). We decided to perform a transabdominal laparoscopic cervicoithsmic cerclage.

Technique: Three trocars, one of $10 \mathrm{~mm}$, two of $5 \mathrm{~mm}$ were used. First step is to proceed to the dissection of the vesicouterine space and isolation of uterine vessel and ureters. A tunnel between the vessels and he cervicoithsmic wall is then made. A vaginal cerclage tape is placed around ithsm and secured with monocryl stich.

At 38 th week a caesarean delivery was performed and the tape was removed

\section{V3_04}

Correction the pelvic floor disorders with hybrid mesh M. Jose Martinez-Paya Hospital de la Ribera, Alzira-Valencia, Spain

Objective: The aim of this video is to present the technique we are using in our center to correct pelvic floor defects in women. We have placed 195 implants from July 2004. In this video we will show the anterior and posterior correction with a new hybrid mesh.

Methods: The technique, after urodynamic study with a pessary in order to avoid possible masked SUI, will start repairing the anterior compartment and afterwards the posterior one. The repair of the anterior compartment will start with a longitudinal incision on the vaginal anterior wall from the fold of the periurethral promontory, due in this case there is no additional incontinence correction technique, to $1 \mathrm{~cm}$. of the reflection with the uterine neck or of the scar in the vaginal vault.

Later, bilateral dissection in paravesical spaces until reaching the tendineous arc of the endopelvic fascia and the ischial spine. Prepared the paravesical spaces, we proceed to prepare incisions over the obturator fossa.

The anterior and posterior edges of the obturator foramen are identified and in order to start with anterior incisions nest to genitocrural fold, being in line at the level of the clitoris. 
Posterior incisions would be at the level of posterior edge of the obturator foramen which varies according to patient anatomy, but it is approximately $2 \mathrm{~cm}$ outside and $3 \mathrm{~cm}$ downwards. The space between the four incisions is really important in order to extend the mesh properly.

Needles with Hook shape and with a snare extension are introduced. After passing the vaginal incision the snare extension must be deployed in order to catch the leg of the mesh, to traction it and to assure it in the needle and to traction towards the superficial incision. We must repeat same procedure with the four arms of the implant. The mesh is manufactured with two layers one of lightweight polypropylene and the other of cross-linked dermis porcine collagen. In the anterior compartment we place the collagen facing the bladder in order to preserve it, and posterior compartment we place the collagen facing the vagina in order to preserve it (we could chose to change it to preserve the vagina or the bladder or the rectum)

The mesh is fixed to the bladder neck and to the vaginal vault or to the uterine isthmus. The vaginal incision is closed with continuous suture.

Afterwards we proceed to repair posterior compartment. We perform a longitudinal incision at the posterior vaginal wall from $1 \mathrm{~cm}$. of the vaginal vault or up to one $\mathrm{cm}$. above the perineal body. Pararectal dissections in order to reach both ischial spines (both sides). Perineal incisions should be performed at $3 \mathrm{~cm}$ lateral and posterior to the anus. Needles with bow-shaped and with a loop extension are introduced. We guide Needle through ischiorectal space parallel to the rectum. We drive needle to $1 \mathrm{~cm}$ from ischial spine and then deploy extension loop to the vaginal incision in order to catch the arm of the hybrid mesh, first superior ones, the ones which reaches ischial spine and afterwards inferior ones which are driven to the perineal body, leaving this way a completed extended mesh, reinforcing rectovaginal fascia. Then we perform traction of the arms, superior and inferior fixation of the mesh, and we finish closing the vaginal incision with continuous suture. We leave a Foley catheter and a vaginal packing is placed in the vagina for $12-24 \mathrm{~h}$. We finish the procedure with a systematic rectal tact.

Results: We have placed 195 implants, which 16 of them where us this new hybrid mesh with good immediate results pre and postoperative and a 3 months follow-up.

Conclusion: The use of these kind of implants seems to be and effective, safe and comfortable which can give good results. Our experience with biological meshes is good and it seems that with this new hybrid mesh we will be able to obtain the benefits of the biological and synthetic meshes.

\section{V3_05}

Treatment and diagnosis of non-tubal ectopic pregnancies
O. Istre, R. Svenningsen

Department of Obstetrics and Gynecology, Oslo, Norway

Objective: Management of non-tubal ectopic gestations remains an inadequately explored clinical field due to the rarity of the presentations. A major disadvantage of twodimensional pelvic ultrasonography is its inability to reconstruct the uterine coronal axis.

Method: Cases presented are interstitial, and C-section scar pregnancy.

Result: The advances in minimal access techniques and imaging modalities have resulted in novel fertility preserving endoscopic procedures. The patients received methotrexate and surgical treatment either initially or delayed surgery with laparoscopy or hysteroscopy.

Conclusion: Treatment with the least invasive method, either by minimal access techniques, non-invasive radiological procedures or medical treatment should be encouraged.

\section{V3_06}

Laparoscopic sacrocolpopexy-standardized technique from a prospective trial on 101 patients

D. Sarlos, LaVonne Kots, G. Schär

Department of Gynecology Kantonsspital Aarau, Aarau, Switzerland

Introduction: From 2003 till 2007 we performed a prospective clinical study evaluating laparoscopic sacrocolpopexy focusing on objective anatomical results and postoperative quality of life. Pre- and postoperative data were collected using the prolapse quantification system (POP-Q) and the Kings Health questionnaire.

Study results: One hundred one patients completed the study with a median follow-up of 12 months. The subjective cure rate was $93 \%$ the objective cure rate according to the International Continence Society (ICS) classification of prolapse was $98 \%$. The most severe intraoperative complications were four bladder and three rectal lesions. The main site of objective recurrence was the anterior compartment with $6 \%$ recurrences of which only $2 \%$ were symptomatic. No apical recurrences occurred. There was a significant postoperative improvement in overall quality of life and in sexual quality with less than $1 \%$ de novo dyspareunia. No vaginal mesh erosion occurred in this short time follow-up.

Video content: The video shows the intraoperative setup, the instruments used, the position of the surgical team, the specially designed vaginal retractor and the surgical technique we used as a standard for the prospective study described above. Especially the posterior dissection to the levator any muscle where the posterior mesh was attached and the anterior sub-vesical dissection where the anterior mesh was attached are well illustrated. The video also 
shows the technique of laparoscopic suturing in the deep pelvis or the promontory as all fixations were performed with laparoscopic sutures, without using any tackers or staplers.

V3_07

Laparoscopic resection of rectal endometriosis: how to prevent postoperative rectovaginal fistulae by using fat tissue of pararectal space

H. Roman, J.J. Tuech, I. Chanavaz-Lacheray, L. Marpeau University Hospital, Rouen, France

Introduction: Laparoscopic resection of rectal endometriosis is usually associated with the removal of the vaginal posterior fornix because of its frequent infiltration by the disease. Consequently, there is a high postoperative risk of rectovaginal fistula, due to the occurrence of an abnormal communication between the colorectal anastomosis and the vaginal wound. In order to prevent this postoperative complication, surgeons use to carry out an omentoplasty, by the section of the omentum along the inferior board of the transversal colon followed by its placing between the two wounds. However, the omentoplasty is a supplementary stage of the surgical procedure and increases the operative time. Furthermore, the section of the omentum along the colon may require to change the placement of the surgical team and of the endoscopic video tower, the change of the operative field from the pelvis to the upper quadrants of the abdomen. We suggest a safe, rapid and easier procedure to prevent rectovaginal fistulae using the fat tissue depending on the mesorectum and pararectal space.

Methods: The dissection of the rectal endometriosis nodule usually requires to open the pararectal space down to the lateral limits of the nodule. During this stage, the preparation of a flap of fat tissue depending on the pararectal space makes sure of preserving a posterior attachment on the mesorectum.
At the end of the procedure, the flap is placed between the vaginal wound and the colorectal anastomosis, and fixed by two separate stitches.

Results: Five women have benefited from this technique from January 2008 to June 2008. All women presented with rectal endometriosis involving the posterior fornix of the vagina and benefited for segmental resection of the rectum followed by immediate colorectal anastomosis. We did not encounter any difficulty to perform the "fat tissue-plasty" technique in less than five minutes on average. No rectovaginal fistula was recorded.

Conclusion: Fat tissue located in the pararectal space might be use to replace an omentoplasty during laparoscopic removal of rectal endometriosis. This technique appears to be quick and safe, and it could be as efficient as the omentoplasty in the prevention of postoperative rectovaginal fistula.

\section{V4_01}

An alternative method for removal of bulky uterus after total laparoscopic hysterectomy

J. English, J. Lo

Worthing Hospital, Worthing, UK

We wish to show a procedure as an alternative to using a morcellator to reduce the size of a bulky uterus found to be too big to pass through the vagina. We performed total laparoscopic hysterectomy for a patient with a big uterus due to adenomyosis. We used a long bladed knife which was passed directly through the anterior abdominal wall in the midline of suprapubic region to cut the uterus into four or more portions. These smaller portions of the cut uterus were then removed through the vagina for histopathology. We kept pneumoperitoneum throughout the procedure by using a sanitary pad held tightly against the vulva. Our experience has shown this can be performed without major complications in a cost-effective manner. 